\title{
Experimental and numerical research of synthetic jet array
}

\author{
Petra Dančová ${ }^{1,2, a}$, Jan Novosád ${ }^{1}$, Tomáš Vit ${ }^{1}$ and Zdeněk Trávniček ${ }^{2}$ \\ ${ }^{1}$ Technical University of Liberec, Studentská 2, 46117 Liberec 1, Czech Republic \\ ${ }^{2}$ Institute of Thermomechanics, CAS, v.v.i., Dolejškova 5, 18000 Praha, Czech Republic
}

\begin{abstract}
This paper describes the additional research of the synthetic jet array in a channel flow and continues the paper of authors Dančová, Trávníček and Vít, [1]. Numerical simulations support the experiments from [1] and the influence of the new slope of the synthetic jet orifices is studied here. This research will be used for preparation of the experiments with inclined orifices of the synthetic jet array.
\end{abstract}

\section{Introduction}

This work continues a larger group of experiments focused on the research of the synthetic jet (SJ) array interacting with a laminar channel flow [1 and 2]. This arrangement can be used in micro-scale application (e.g. a cooling of micro-electronics). In micro dimensions the flow is usually laminar with very small Reynold numbers. Therefore, the SJ actuator or the SJ array can be successfully used to disturb a laminar flow in these conditions.

Characteristics, parameterization and application of synthetic jet are described e.g. in [3-5]. Briefly described, $\mathrm{SJ}$ is generated by the periodical motion of an actuator oscillating membrane. In first half of the period of the membrane movement, the fluid is sucked into the sealed actuator cavity through the orifice. In the second half of the membrane movement, this fluid is extruded from the actuator cavity. The vortex rings are formed at the lip of the orifice during the fluid extrusion. These rings should move high enough from the orifice to prevent suction back into the cavity during the suction phase of the time period.

Based on the experimental results from [1], the adjustment for the numerical simulation in sw Ansys Fluent was prepared and tested. This adjustment will be used for new experiments preparation with inclined orifices of the synthetic jet array.

\section{Experimental setup and method}

\subsection{Synthetic jet array experimental setup}

The SJ array is made from four SJ actuators placed across the channel cross section. The actuator is made from Plexiglas and consists of a sealed cavity equipped with an emitting orifice and with a periodically moving piezoelectric membrane KINGSTATE KPS-100.

\footnotetext{
${ }^{\mathrm{a}}$ Corresponding author: petra.dancova@tul.cz
}

Diameter of an actuator cavity is $36 \mathrm{~mm}$; the cavity height is $5 \mathrm{~mm}$.

For our research two kind of geometry for SJ actuators orifices were designed. Firstly, the orifices were placed upwards along the cavities axes and have diameter and height of $3 \mathrm{~mm}, 5 \mathrm{~mm}$ respectively (see figure 1). In the second geometry type, the orifices were inclined of 45 degrees from the SJ actuator axes (figure 2).

SJ array is placed on the Plexiglas with dimensions: length (length with start) $\mathrm{x}$ width $\mathrm{x}$ thickness $=(860(880)$ $\times 200 \times 10) \mathrm{mm}$. The wall inlet was carefully rounded, and the SJ array was placed $520 \mathrm{~mm}$ from the beginning. The wall was put into the circulation water channel, which is described in [1 and 2].

\subsection{Experimental method - PIV}

The particle image velocimetry (PIV) method was used for the experiments. In this a non-contact laser optical method for research of flow, turbulence, microfluidics, and many others processes, where the measurement fluid has to be transparent enough. In the PIV, the velocity vectors are derived from sub-sections of the target area of the particle-seeded flow by measuring the movement of particles between two light pulses. For more description of this method see [6-9].

The settings of the PIV system was set the same as in [1]: a unit "Timer box" and DynamicStudio v.2.30 (DANTEC) software were used. All the control boards of the lasers, cameras, and timers of the input and output signals were placed directly into the main computer. DynamicStudio is used as a control and processing program for the unit Timer box. The computer takes the role of a measuring center. Data communication between the PC, laser, and cameras runs through the internal protocols of the DynamicStudio control software and are impossible to modify. Starting signals are taken out by means of a National Instruments PCI-6601 counting 
measuring card into the unit Timer box with eight BNC connector outputs and two inputs.

Signals for the start of every flash lamp and Qswitching of the laser are led through one cable, whose position it is necessary to define - to Timer box to connect the starting trigger signal into two inputs.
Figure 3 shows the principle of basic synchronization. From the SJ signal generator, the TTL signal goes into the PC (Timer box). The laser and camera start with the leading edge of the TTL. The starting point of measurement is changed during the SJ period and, therefore, the whole period is measured.

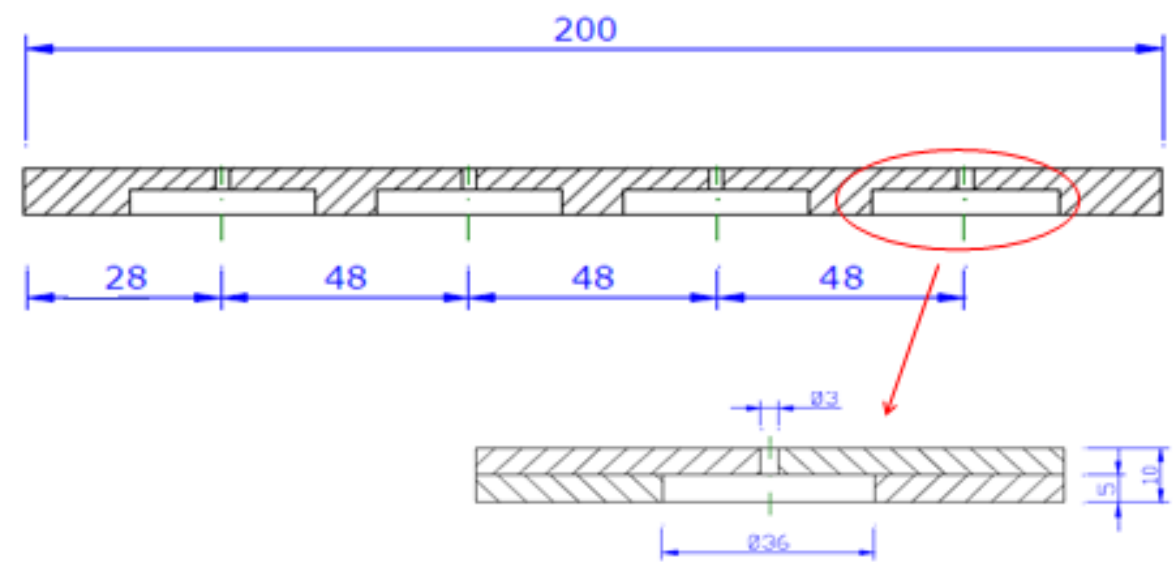

Figure 1. Schematic view of SJ array (straight orifices).

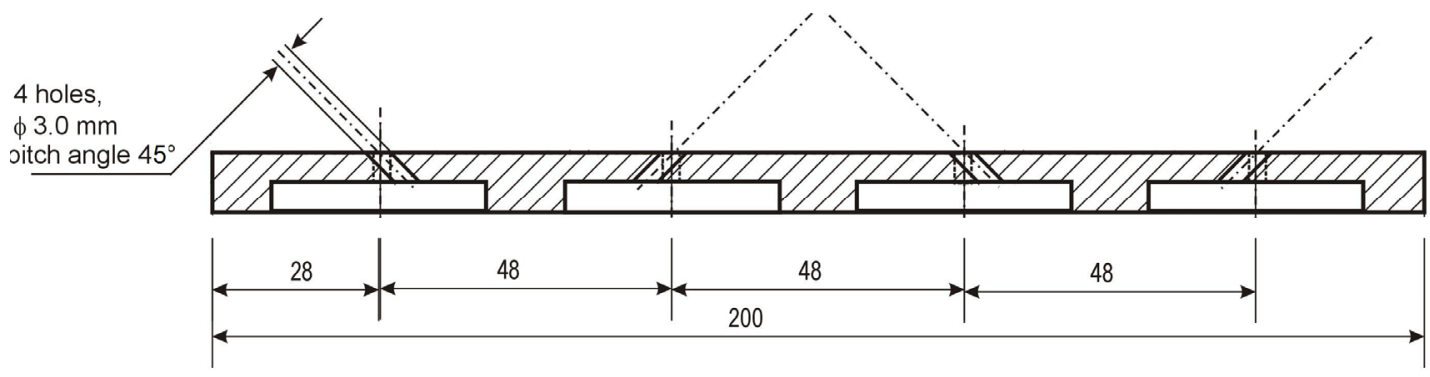

Figure 2. Schematic view of SJ array (inclined orifices).

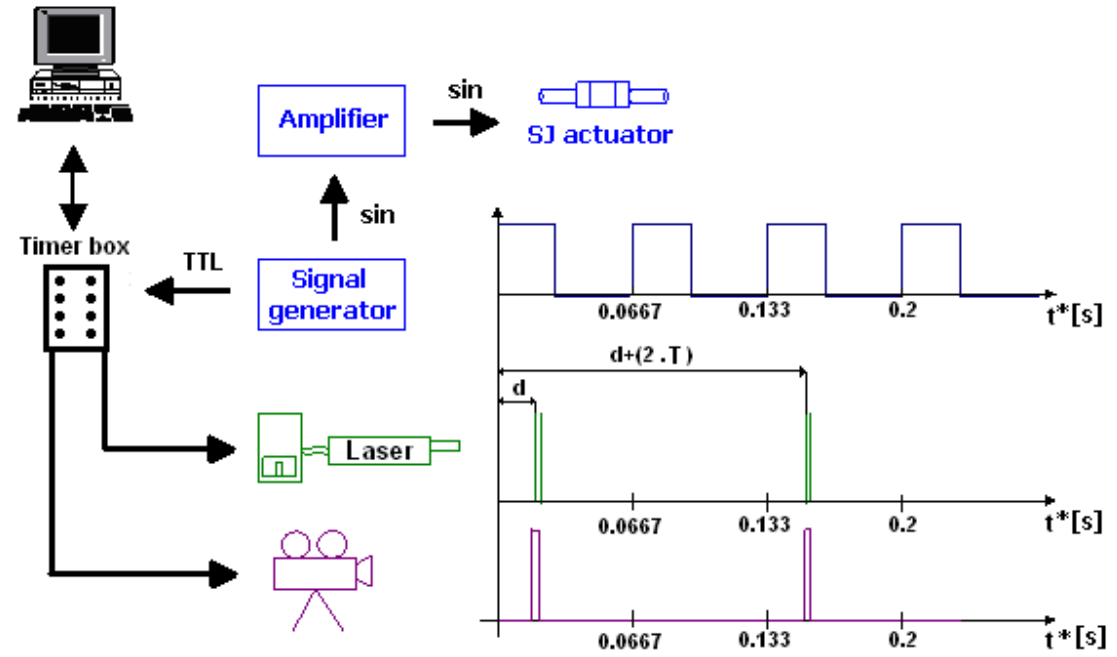

Figure 3. Schematic of basic synchronization for PIV measurements (T means the period).

The delay inside the electronic synchronization devices does not exceed $3 \mathrm{~ms}$.

Polyamide balls with diameters of $20 \mathrm{~mm}$ (DANTEC) were used as the seeding particles. The particles were illuminated by a double pulse laser with a maximum
$125 \mathrm{~mJ}$ per $10 \mathrm{~ns}$ pulse at a repetition rate of $2 \times 15 \mathrm{~Hz}$. The typical delay time between two pulses was $5 \mathrm{~ms}$.

The laser beam was expanded into a light sheet of approx. $1 \mathrm{~mm}$ thick. The image pairs were acquired using 
the HiSense MKI camera (DANTEC) with a spatial resolution of $1280 \times 1024$ pixels.

The resulting vector maps were averaged over 100 PIV records. Velocity vectors were determined by adaptive correlation using interrogation windows of $32 \times 32$ pixels at a $25 \%$ overlap. Data processing was done using DynamicStudio 2.21 commercial software (DANTEC).

\section{Numerical modelling}

Numerical simulations were realized to promote the results of experiments. Preparation of model and mesh was carried out in Ansys Workbench environment. Ansys Design Modeler was used to create 3D model.

\subsection{Model geometry and mesh}

The model was based on the geometry of the SJ array. Each model presents only a half of the SJ array, because the symmetry boundary condition was used to simplify the simulations. Close region around the SJ array were modelled. Two models of channel were created as a tested area for both cases - straight and inclined SJ actuators orifices. Final models with basic dimensions are shown in figure 4 .
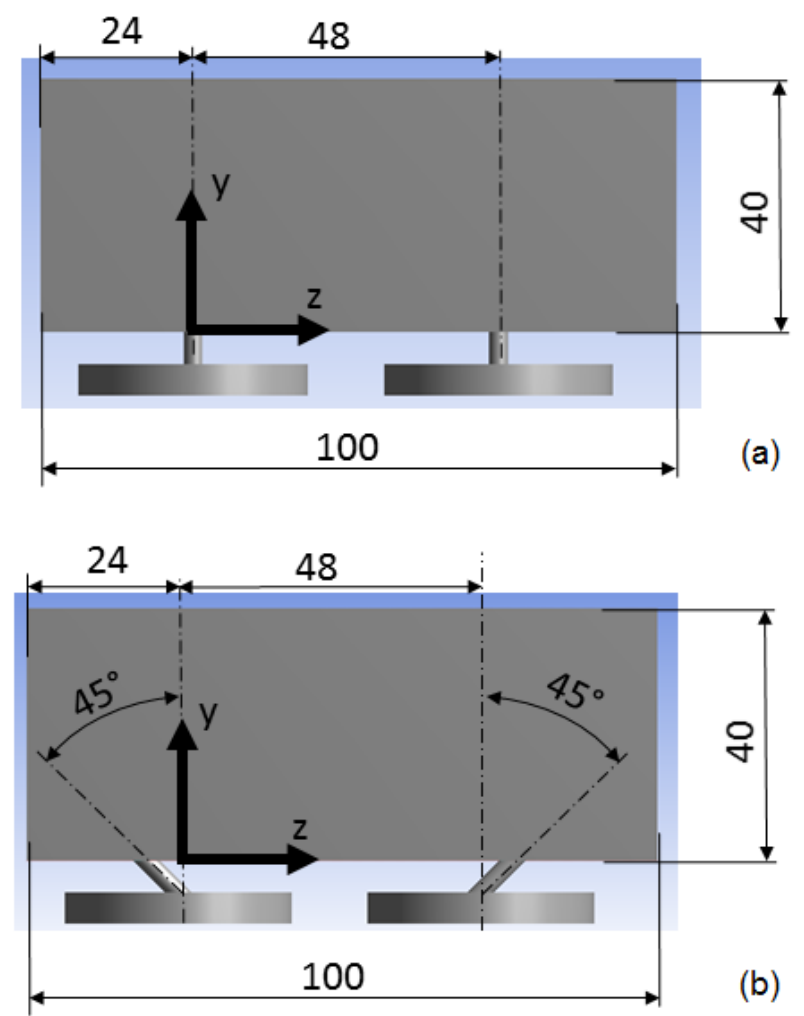

Figure 4. Model of SJ array - front view, (a) straight SJ orifices, (b) inclined SJ orifices.

Computational meshes for each model were generated in ANSYS Meshing. Automatic functions were used to create hybrid mesh with predominant hexahedral elements. Both meshes contain around 950000 elements.

\subsection{Boundary conditions}

Boundary conditions are shown in figure 5. On unlabelled faces, the boundary condition „wall“ is applied. On the membrane faces, the BCs ,,velocity-inlet“ are applied. Oscillation of membranes is modeled with UDF function, which is defined as dependency of velocity on time (following equations and methodology in [2]). For the description of parameters see table 1.

Table 1. Boundary conditions.

\begin{tabular}{|c|c|l|}
\hline Label & BC type & Parameters, description \\
\hline Inlet & velocity-inlet & Inlet velocity $0.0067 \mathrm{~m} / \mathrm{s}$ \\
\hline Outlet & pressure-outlet & Channel outlet \\
\hline Membranes & velocity-inlet & $\begin{array}{l}\text { Velocity profile defined } \\
\text { by UDF function }\end{array}$ \\
\hline Symmetry & symmetry & Symmetry plane \\
\hline Wall & wall & Outer walls of channel \\
\hline
\end{tabular}

\subsection{Methodology of results assessment}

To obtain velocity fields in close area to the SJ array several cutting planes were defined (for description see table 2) Global coordinate system is defined by triad X, $\mathrm{Y}, \mathrm{Z}$ (see figure 4). To resolute the actuators, the actuator which is closer to symmetry plane is called „inner“ actuator, the another one is called „outer“ actuator.

Table 2. Cutting planes.

\begin{tabular}{|c|l|}
\hline Label & \multicolumn{1}{|c|}{ Parameters, description } \\
\hline SJ-cross & Equal to $\mathrm{YZ}(\mathrm{x}=0)-$ through all actuators \\
\hline In-PL & Equal to $\mathrm{XY}(\mathrm{z}=0)-$ through inner actuator \\
\hline Out-PL & $\begin{array}{l}\text { Parallel to } \mathrm{XY}(\mathrm{z}=48 \mathrm{~mm})-\text { through outer } \\
\text { actuator }\end{array}$ \\
\hline
\end{tabular}

Velocity fields are obtained using Ansys FLUENT postprocessing tools, especially contours of velocity magnitude and velocity vector fields.

\section{Results}

\subsection{Straight SJ array orifices in a channel flow}

Experiments of the straight SJ array with affection of the laminar channel flow are described in [1 and 2]. The delay between the two laser pulses was $t=5 \mathrm{~ms}$. The working frequency of SJ actuators was $f=15 \mathrm{~Hz}$. Experimental results shown in figure 6 were performed in the plane $x-y$, for $z=(0$ and 48$) \mathrm{mm}$ according to the geometry used in figure 4 . The results plotted in figure 6 are in the form of velocity magnitude contours (in discrete drawing style) in time $t / T=0.75$ (maximum extrusion velocity). There is visible small bending of a SJ (approx. $10^{\circ}$ ) and $\mathrm{SJ}$ reached the opposite wall $(y=40$ $\mathrm{mm}$ ) at the location of approx. $x=8 \mathrm{~mm}$. The time-mean velocity through the channel was evaluated approx. $U_{\mathrm{C}}=0.0067 \mathrm{~m} / \mathrm{s}$. The synthetic jets' time-mean orifice velocity as $U_{0}=0.06 \mathrm{~m} / \mathrm{s}$. These velocities are entered as the boundary conditions for the numerical simulations of this problem. 


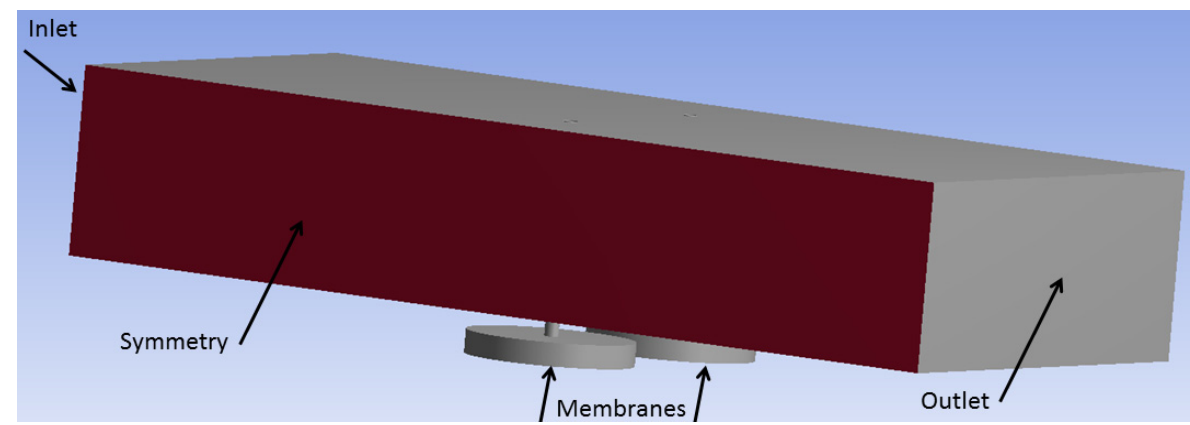

Figure 5. Boundary conditions used in numerical modelling.
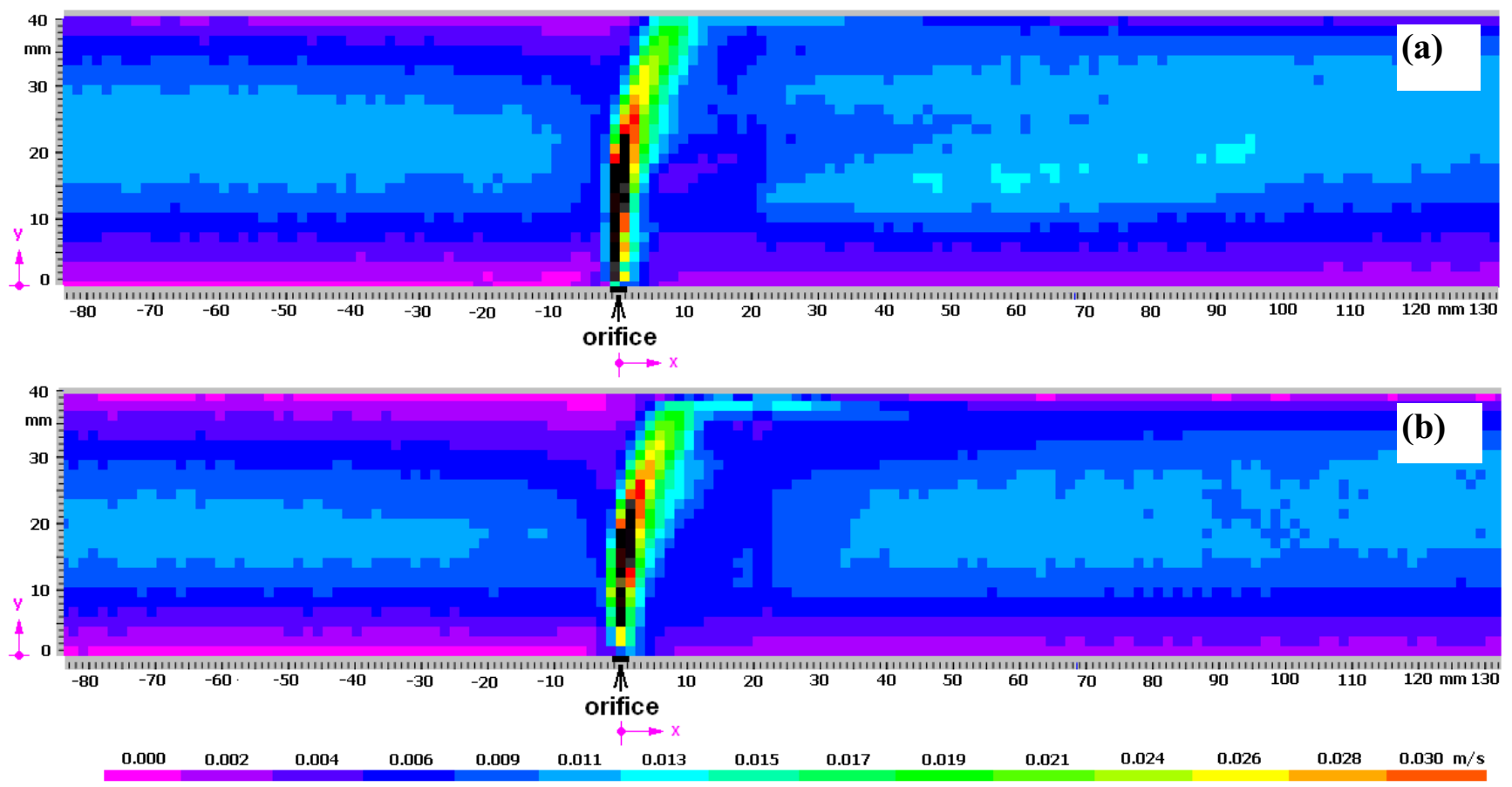

Figure 6. Straight SJ orifices - the experimental results (plotted in discrete drawing style), $t / T=0.75$,

(a) outer SJ actuator, (b) inner SJ actuator.
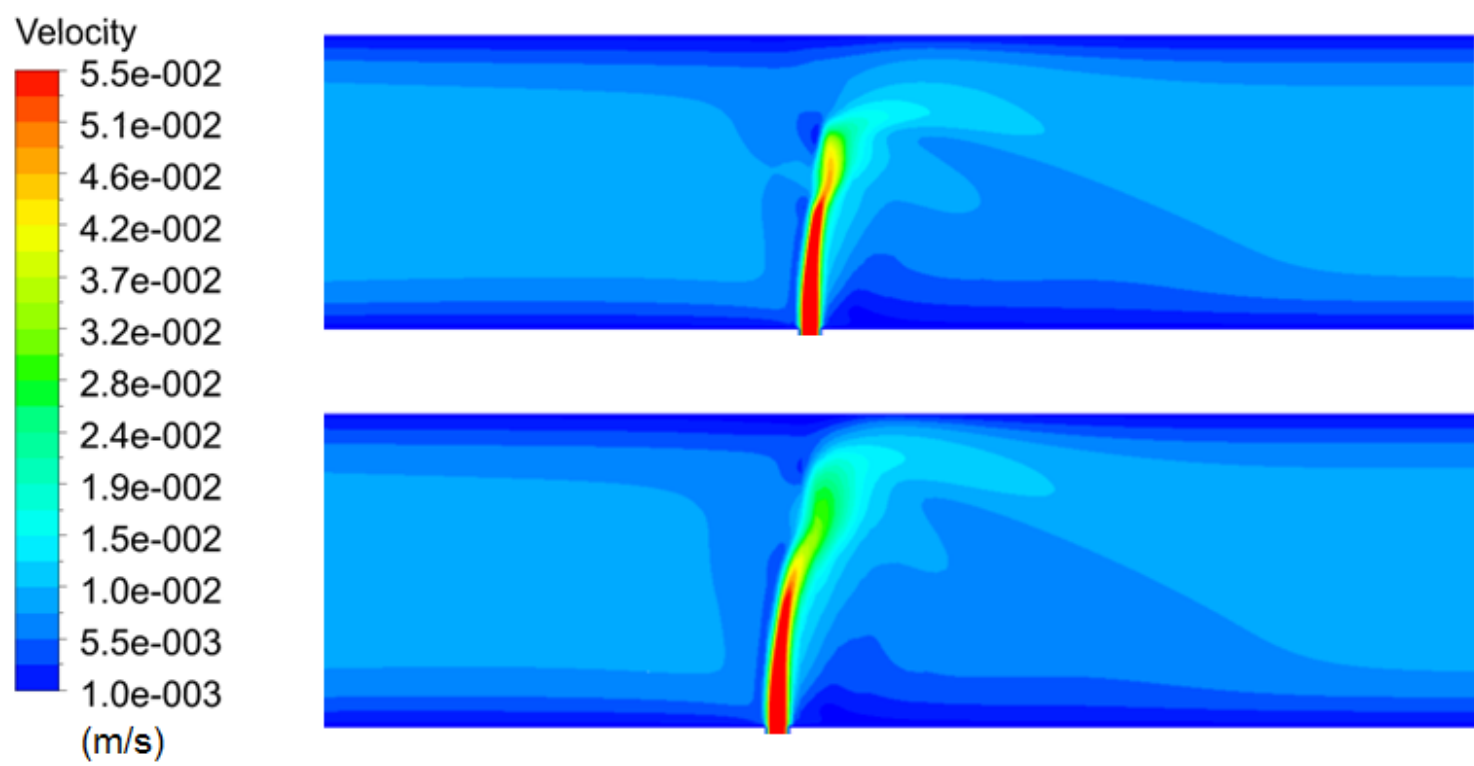

(a)

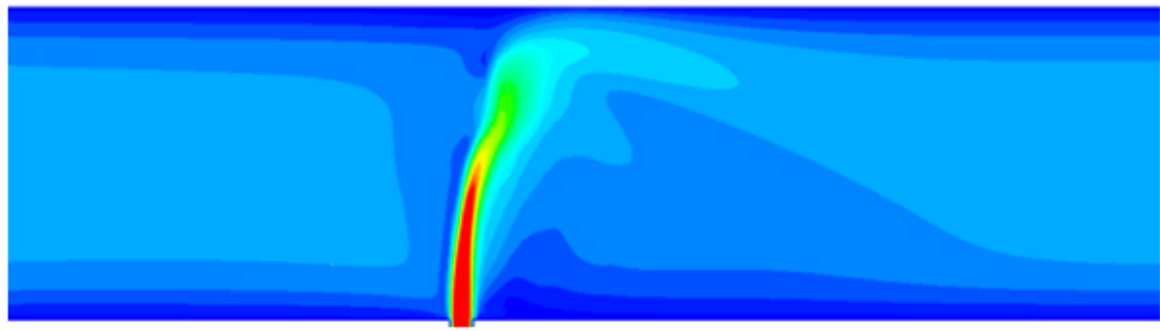

(b)

Figure 7. Straight SJ orifices - results of the numerical simulation, $t / T=0.75$, (a) outer SJ actuator, (b) inner SJ actuator. 
Figure 7 shows the numerical simulation of the same situation as is in the experiments: working frequency of SJ actuators was $15 \mathrm{~Hz}$, the outer and inner SJ actuator was in the maximum extrusion time $(t / T=0.75)$. Comparison of the experiment and the numerical simulation in the plane $x-y$ shows very good agreement (see figure 6 and 7 ).

The water channel arrangement used in [1 and 2] did not enable to measure the situation in the plane $x-z$. To make visible the behaviour of the SJ array in the channel flow in the plane $x-z$ see figure 8 . This figure shows the contours of velocity and velocity vector fields in time $t / T=0.25$ and 0.75 , i.e. maximum suction velocity and maximum extrusion velocity, respectively. Because the velocity in the SJ orifices is one order higher than the velocity in a channel, two scales are used in this figure. Figure 8 also shows detailed view into the SJ actuators. This view was not possible to investigate experimentally.
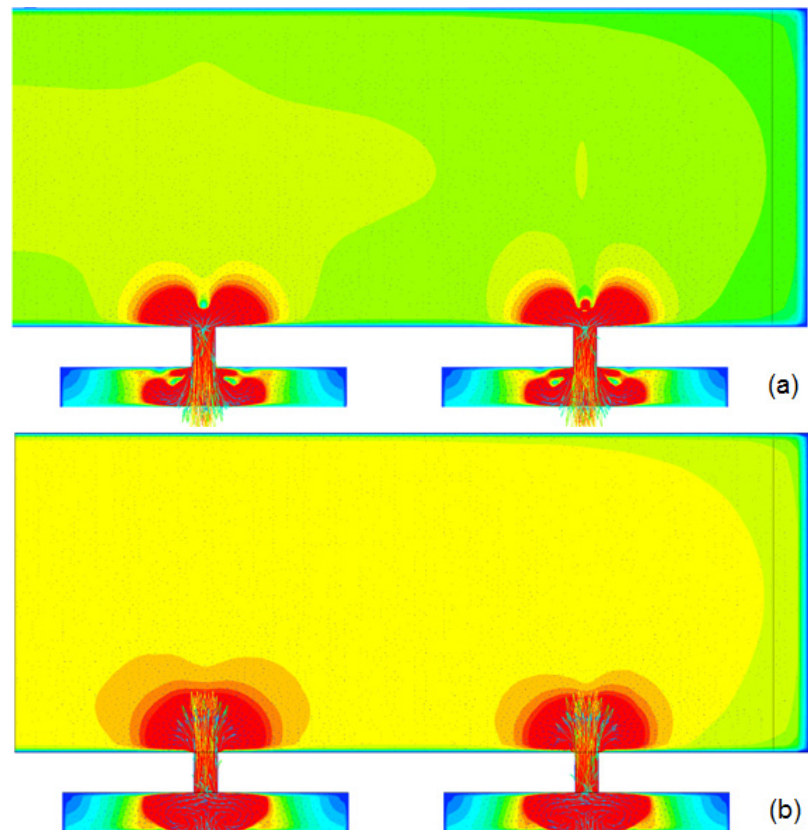

Velocity
\begin{tabular}{|c|}
\hline $1.0 \mathrm{e}-002$ \\
$9.3 \mathrm{e}-003$ \\
$8.6 \mathrm{e}-003$ \\
$7.9 \mathrm{e}-003$ \\
$7.1 \mathrm{e}-003$ \\
$6.4 \mathrm{e}-003$ \\
$5.7 \mathrm{e}-003$ \\
$5.0 \mathrm{e}-003$ \\
$4.3 \mathrm{e}-003$ \\
$3.6 \mathrm{e}-003$ \\
$2.9 \mathrm{e}-003$ \\
$2.1 \mathrm{e}-003$ \\
$1.4 \mathrm{e}-003$ \\
$7.1 \mathrm{e}-004$ \\
$0.0 \mathrm{e}+00$
\end{tabular}

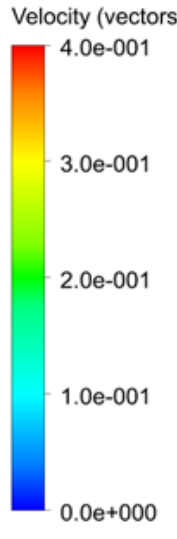

$(\mathrm{m} / \mathrm{s})$

$(\mathrm{m} / \mathrm{s})$

Figure 8. Straight SJ orifices - numerical simulation,

(a) $t / T=0.25$,

(b) $t / T=0.75$.

experiment, the same adjustment for numerical simulation for inclined SJs was used.

Figure 9 shows the affection of inclined SJs with the channel flow in time of maximum suction and extrusion velocity $(t / T=0.25$ and 0.75$)$ in plane $x-z$. The SJ actuators working frequency was $15 \mathrm{~Hz}$. Figure 9 also shows velocity distribution inside the SJ actuator.
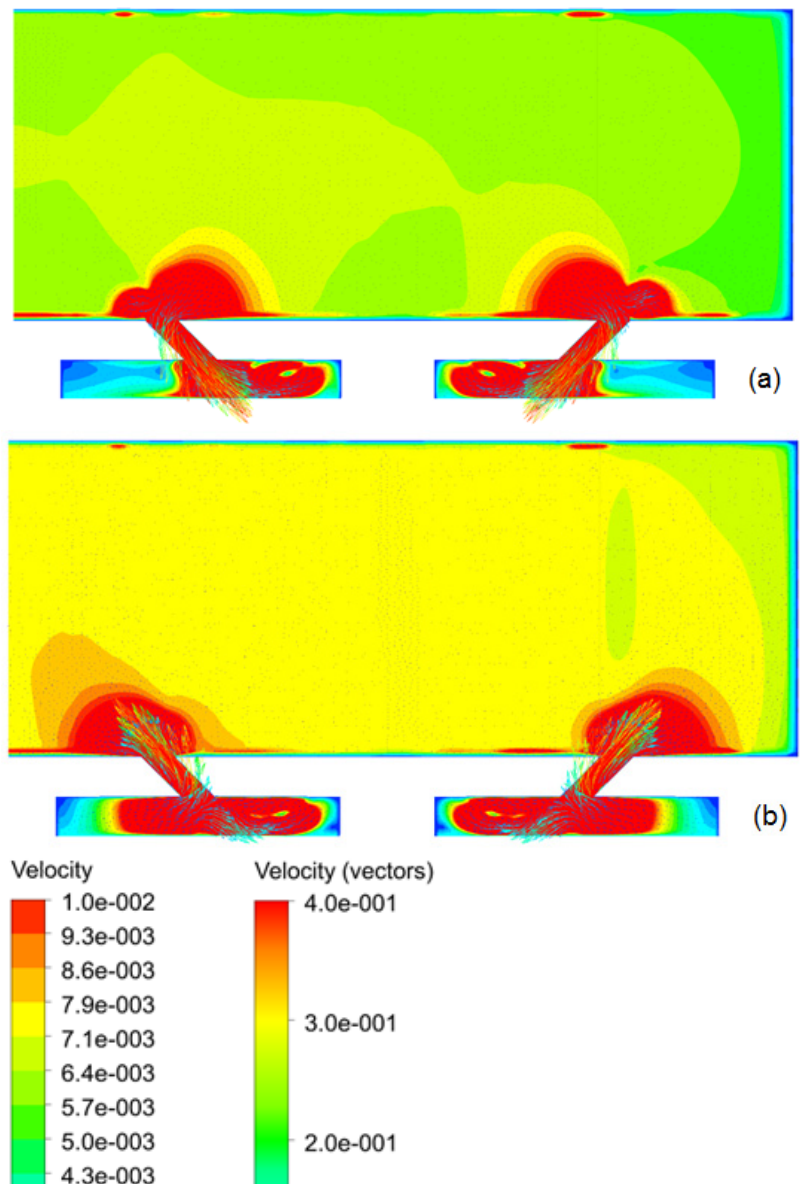

Figure 9. Inclined SJ orifices - numerical simulation,

$1.4 \mathrm{e}-003$

$7.1 \mathrm{e}-004$

$0.0 \mathrm{e}+000$

(a) $t / T=0.25$

$(\mathrm{m} / \mathrm{s})$

$(\mathrm{m} / \mathrm{s})$

(b) $t / T=0.75$

For the experimental investigation - to set the laser sheet in a right position, the figure 10 will be used. This figure makes visible what is happened in a symmetry plane (a), how the inner and outer actuator influence a channel flow in the plane $x-z$ (b), (c), and also shows behaviour of a channel flow between the inner and outer SJ actuator (d). The SJ orifices were plotted in time of maximum extrusion $(t / T=0.75)$.

\section{Discussions}

\subsection{Inclined SJ array orifices in a channel flow}

Because the results from the numerical simulation of the straight SJ orifices are in very good agreement with the

This paper brings results of a research of a channel flow influenced with a SJ array. According to the experiments from [1 and 2], the numerical simulations were carried out. Experimental data and results from [1 and 2] were 
used for adjustment of geometry, numerical model and boundary conditions. After a successful comparison, this model was used for next step of our research - for inclined SJ actuator orifices investigation and these results will be used to prepare the experiments.
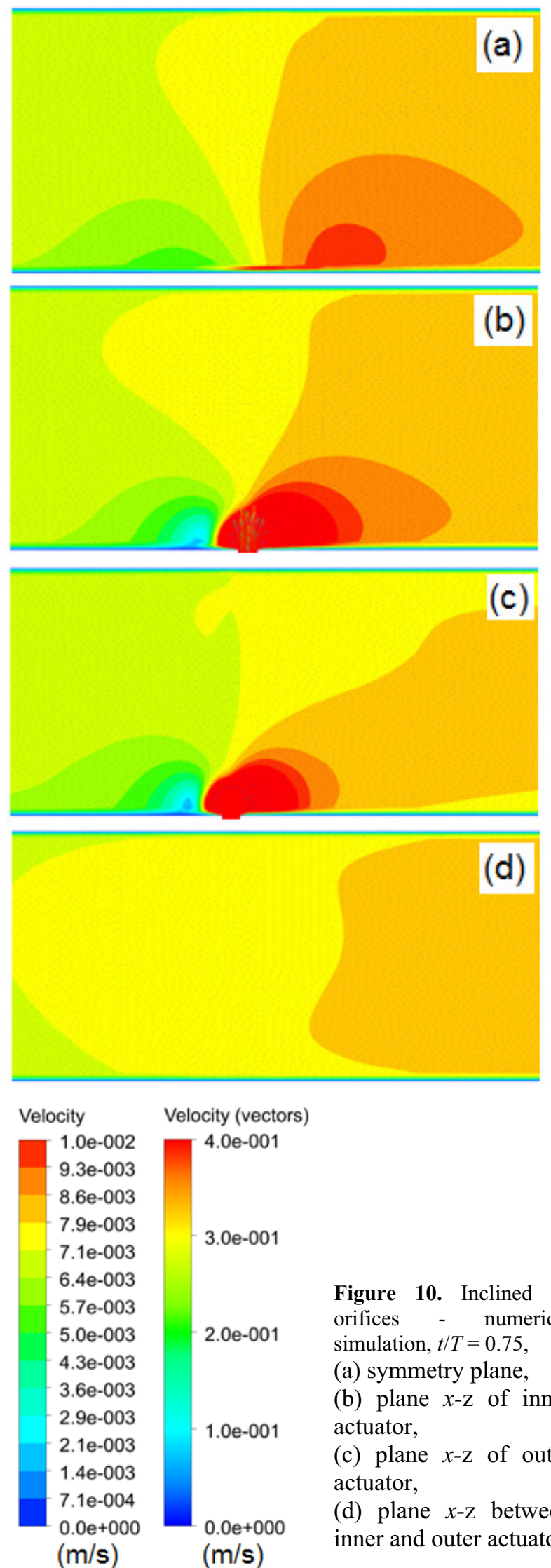

Figure 10. Inclined SJ orifices - numerical simulation, $t / T=0.75$,

(a) symmetry plane,

(b) plane $x$-z of inner actuator,

(c) plane $x-z$ of outer actuator,

(d) plane $x-z$ between inner and outer actuator.

\section{References}

1. P. Dančová, Z. Trávníček, T. Vít, [Experimental investigation of a synthetic jet array in a laminar channel flow,] EPJ Web of Conferences, Vol. 45, 01002 (2013)

2. P. Dančová, Ph.D. thesis (Technical university of Liberec, 2012)

3. B.L. Smith, A. Glezer, Phys. Fluids 10, 2281 (1198)

4. J.E. Cater, J. Soria, J. Fluid Mech. 472, 167 (2002)

5. A. Glezer, M. Amitay, Annu. Rev. Fluid Mech. 34, 503 (2002)

6. http://www.lavision.de/en/techniques/piv.php

7. http://www.tsi.com/Fluid-Mechanic-Systems/

8. http://www.dantecdynamics.com/particle-imagevelocimetry

9. http://www.openpiv.net/

10. S. Gavlas, P. Durčanský, R. Lenhard, J. Jandačka, [Mathematical simulation of heat exchanger working conditions,] EPJ Web of Conferences, Vol. 92, 02019 (2015)

11. M. Kasanický, R. Lenhard, K. Kaduchová, M. Malcho, [An optical method for measuring the thickness of a falling condensate in gravity assisted heat pipe,] EPJ Web of Conferences, Vol. 92, 02031 (2015)

\section{Acknowledgements}

We gratefully acknowledge the support of the Grant Agency of the Czech Republic - Czech Science Foundation (project no. 14-08888S). 